\title{
Development of a Lab-on-a-Chip for Detection of Bacteria in Food Samples
}

\author{
Dibyo Sarkar ${ }^{1}$, Iqbal Jamal ${ }^{2}$, Sushanta K. Mitra ${ }^{* 1}$ \\ ${ }^{1}$ Micro Nano-scale Transport Laboratory, Department of Mechanical Engineering, University of \\ Alberta, Edmonton, T6G 2G8, Canada, \\ 'All correspondence to this author: Email: sushanta.mitra@ualberta.ca \\ ${ }^{2}$ AQL Management Consulting Inc., 4216 109A St NW, Edmonton, AB T6J2R8, Canada
}

\begin{abstract}
:
We developed an optical biosensor of nanodimensions, for the detection of bacteria in food samples, which has an integrated Mach-Zehnder Interferometer (MZI) configuration. MZI works on the principle of total internal reflection having a sensor arm, where the bacteria is to be bound by biofunctionalization, and a reference arm. The MZI is fabricated on a silicon substrate with silicon nitride (Refractive index $=2.00$ ) acting as the core and silicon dioxide (Refractive index=1.46) acting as the upper and lower cladding. Light from a laser source ( $\mathrm{He}-\mathrm{Ne}$ ) will be coupled into the waveguide having the MZI configuration which is split into the two arms, then after a certain distance, they recombine again. This is done by using a diverging and converging $\mathrm{Y}$-junction, respectively. The design of the MZI has been created using simulations in order to get a monomodal propagation of light with minimal losses. The Y-junctions have been designed so as to allow the divergence and convergence of the propagating light with a $3 \mathrm{~dB}$ split ratio.
\end{abstract}

Key words: Mach-Zehnder Interferometer, silicon substrate, Y-junction, monomode, simulations.

\begin{abstract}
Introduction
Commercially available detection techniques include enzyme-linked immunosorbent assay (ELISA), enzyme-linked immunofluorescence assay (ELFA), fluorescence in-situ hybridization (FISH), polymerase chain reaction (PCR), etc. [1]. But these techniques are not capable of real-time detection of the pathogens. The use of biosensors provides an alternate and viable approach towards fast detection of deadly pathogens in RTE meat products.
\end{abstract}

To this end, label-free optical biosensors are used. Simplicity in fabrication, portability, small footprint, and high sensitivity are a few other advantages that make optical sensors very promising [2-6].

Mach-Zehnder Interferometer (MZI) based label-free optical sensors have been in use since the 1990s [4, 7-9]. Researchers have been working towards the improvement of the sensitivity of $\mathrm{MZI}$ based devices. High sensitivity can be achieved by reducing the optical losses occurring in the sensor and through monomodal propagation of light $[2,4$, 9]. In this paper, we have used simulation techniques to improve the $\mathrm{MZI}$ design and provide an understanding of the dependence of sensitivity of the biosensor on Y-junction dimensions through optical loss analysis.

\section{MZI Working Principle and Configuration}

Label-free optical sensors work on the principle of evanescent field detection. An optical sensor consists of two cladding layers enclosing a core layer. The refractive index of the core layer is higher than the cladding layers. Light travels through an optical sensor due to total internal reflection inside the core layer. During total internal reflection inside the core layer, a small portion of the reflected light penetrates through the core-cladding interface as an electromagnetic field and propagates parallel to the surface, in the plane of incidence. This electromagnetic field is called the evanescent field. The evanescent field is usually $1 / 5$ th of the wavelength of the incident light and decays exponentially in the direction normal to the direction of propagation of light. Any changes on the sensor surface within the evanescent field caused due to the interaction between the bacteria and immobilized antibodies are manifested (as a change in the effective refractive index) in the evanescent field and this information is carried by it to a detection set-up [3-4, 9]. 
MZI consists of two Y-junction couplers connecting a sensor and a reference arm. Incoming light from a laser is split into the two arms which later recombines providing output signal containing information of the changes that have occurred in the sensor arm. The sensor arm is the site where the refractive index change takes place. This causes a phase shift in the sensor arm as compared to the reference arm. The key is in the design of the $Y$-junctions which will not only reduce optical losses during propagation of light waves through the MZI but also prevent cross coupling of signal between the sensor and reference arms.

\section{Experimental Setup}

The schematic of the experimental setup to be used in the biosensing of Listeria monocytogenes using the $\mathrm{MZI}$ configuration is shown in Fig. 1.

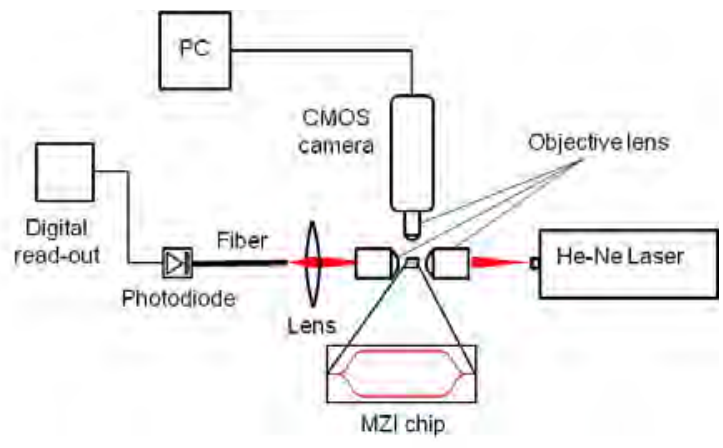

Fig. 1. Schematic of the experimental setup.

Light source from a He-Ne laser $(633 \mathrm{~nm})$ is coupled into the $\mathrm{MZI}$ chip using a $40 \mathrm{x}$ objective lens. Light propagating through the chip is outcoupled into a multimode fiber $(62.6 \mu \mathrm{m}$ core diameter) through a lens assembly. The multimode fiber carries the light into a photodiode for power measurements. The chip is placed on an $X Y Z$ stage (not shown in the figure) with piezo controlled resolution of $20 \mathrm{~nm}$. A CMOS camera, connected to a PC is placed above the chip to simplify the optical alignment of the device.

\section{MZI Design}

As mentioned previously, reducing the losses in an optical biosensor should intuitively increase the sensitivity of the sensor. Intermodal dispersion and intermodal scattering are two major sources of losses. Although scattering losses are inevitable due to imperfections during fabrication, intermodal dispersion losses can be nullified by using single-mode (monomodal) propagation of light. Monomodal propagation essentially means that no higherorder modes except the fundamental (or the lowest-order) mode can pass through the sensor. During bio-interaction in the sensor arm, transfer of power from the fundamental mode to higher order modes occurs due to the change of phase. However, since higher order modes are not allowed to form in a monomodal configuration, this power will be radiated out of the sensor thereby creating a relation between phase change $(\phi)$ and output power $\left(P_{\text {out }}\right)$ of the biosensor. They are related as:

$$
P=[1+\cos (2 \phi)] / 2
$$

where,

$$
\phi=(2 \pi / \lambda) \Delta n_{e f f} \ell
$$

Where $\mathrm{P}$, called the normalized output power, is the ratio of the output and input power $\left(P_{\text {in }}\right), \lambda$ is the wavelength of incident light, $\ell$ is the length where the biointeraction takes place in the sensor arm, and $\Delta n_{\text {eff }}$ is the effective refractive index change caused by the biointeraction.

An optical sensor consists of a core and two cladding layers. Light propagates through the core due to total internal reflections. The core is etched to a certain depth in order to establish the MZI configuration (see Fig.2). This is done using photolithography. The height and width of the rib structure forming the $\mathrm{MZI}$ configuration should be very low in order to allow monomodal propagation of light.

The most challenging section of the $M Z I$ is the Y-junction. It needs to be such that light is equally split into the two arms in order to produce highest contrast in the interference signal. Intuitively this means that a $3 \mathrm{~dB}$ split ratio need to be maintained while designing the Y-junction which in turn means a symmetrical structure. This point is further supported by the analysis done by Syms et al. [10].

The distance between the two arms is denoted as $d$ and the length of each arm is $L$ (see Fig. 2 ). If $a_{\text {in }}$ and $a_{\text {out }}$ are the input and output amplitudes of the propagating mode, and $E(x, z)$ is the transverse field direction of the incident light, Syms [10] suggests that for a symmetrical Y-junction, the total transverse field just before the biointeraction $\left(E_{\text {initial }}\right)$ area is:

$E_{\text {initial }}=\left(a_{\text {in }} / \sqrt{2}\right) E_{S}+\left(a_{\text {in }} / \sqrt{2}\right) E_{R}$

where,

$$
\begin{aligned}
& E_{S}=E(x+d / 2, z) \\
& E_{R}=E(x-d / 2, z)
\end{aligned}
$$

$E_{S}$ and $E_{R}$ are the transverse field distribution patterns of the sensor and reference arm, respectively. 
Eq. (3) denotes that each arm has an amplitude $1 / \sqrt{ } 2$ times the input amplitude which means they carry half the power. This reinstates the fact that symmetrical $Y$-junctions display a $3 \mathrm{~dB}$ split ratio.

The total transverse field just after the biointeraction and after a phase change of $\phi$ is given as $\left(\mathrm{E}_{\text {bio }}\right)$ :

$$
E_{\text {bio }}=\left(a_{i n} / \sqrt{2}\right) E_{S} \cdot \exp (j \phi)+\left(a_{i n} / \sqrt{2}\right) E_{R}
$$

Another important design consideration is the bend radius $(R)$ or the angle of bend $(\theta)$ of the $\mathrm{Y}$-junctions. Bending causes additional losses to the scattering losses and should be minimized. The best way to do this is keep the bend radius high or the angle of bend very low.

\section{Simulation Results}

In order to find out optimal $\mathrm{MZI}$ configurations relating to lowest losses, parameters $R, \theta$ and $d$ need to be calculated. This has been done through simulations using RSOFT CAD ${ }^{T M}$, a photonic design software, by RSoft Design Group, Inc. Simulations were performed using a correlation function that correlates the input field $\varphi_{\text {in }}(x)$ and the propagating field $\varphi(x, z)$ to compute all the modes in the waveguides with varying dimensions of the rib structure.

The governing equation used in the simulations is

$$
P(z)=\int \varphi_{\text {in }}(x) \varphi(x, z) d x
$$

where,

$$
\begin{aligned}
& \varphi_{i n}(x)=\sum_{m} A_{m} \varphi_{m(x)} \\
& \varphi(x, z)=\sum_{m} A_{m} \varphi_{m(x)} \exp (i \beta z) \\
& \beta=n_{\text {eff }} \cdot 2 \pi / \lambda
\end{aligned}
$$

Here $P(z)$ is correlation function, $\beta$ is the propagation constant, $\mathrm{n}_{\text {eff }}$ is the effective refractive index and $\lambda$ is the wavelength of incident light. The Fourier coefficient is represented by $A$. The input and propagating fields have the form of a Fourier series. Therefore a Fourier transform of the correlation function is performed while solving for the modal fields. The $x$ and $z$ co-ordinate axes are defined in Fig. 2. The length of the entire design (chip) is $30 \mathrm{~mm}$.

Simulations were performed on two different geometries: straight arms with an opening angle $\theta$ referred as the angular $\mathrm{Y}$-junction; an $S$-bend of radius $R$ referred as the Sbend $Y$ junction (see Fig. 2). All simulations were done with the distance between the two arms (d) not more than $100 \mu \mathrm{m}$, since the arms need to be close to each other [9].
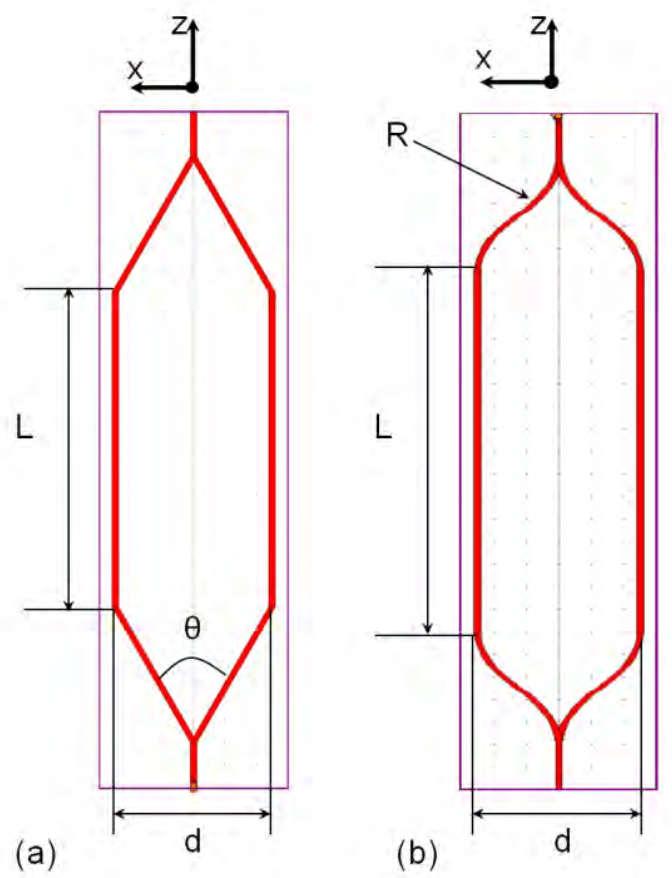

Fig. 2. (a) Angular Y-junction, (b) Sbend Y-junction. On performing simulations using different values of $\theta$ and $R$ in the angular and Sbend $Y$ junction structures, respectively, it was seen that for the same interaction length (L), the Sbend showed less optical losses than the angular structure. For example, for a $22 \mathrm{~mm}$ arm length, the Sbend structure showed losses of around $4 \mathrm{~dB} / \mathrm{mm}$ whereas the angular structure showed $5.6 \mathrm{~dB} / \mathrm{mm}$. The simulation results of an Sbend $Y$-junction with $R=125 \mathrm{~mm}$ are shown in Fig 3. The color bar in part (a) and the $x$-axis of part (b) both show the intensity of the field (1 being the highest and 0 being the lowest). It can be seen that the total loss is about $2.4 \mathrm{~dB} / \mathrm{mm}$ and that the converging $\mathrm{Y}$-junction creates more losses than the diverging $\mathrm{Y}$-junction.

Consequently, it was found that the best results corresponded to $\theta$ values below $2.5^{\circ}$ and $R$ values above $15 \mathrm{~mm}$. For other values, the losses were too high for high sensitivity measurements.

Decreasing $\theta$ to a much lower value and increasing $R$ to a much higher value is constrained by the size of the chip. Since such extreme values would cause either the entire length of the chip to be lot longer or the arm length $(L)$ to be a lot shorter, both of which is 
unacceptable. One solution is to decrease $d$ and bring the arms closer. However, the arms cannot be brought closer than $70 \mu \mathrm{m}$ as cross coupling of signal between the two arms can occur.
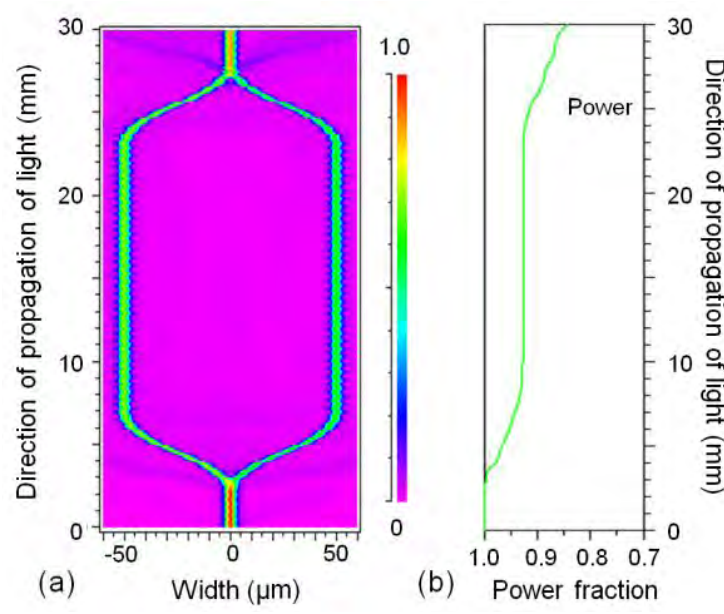

Fig. 3. (a) Transverse electric field through the entire length of the $M Z I$ configuration $(R=125 \mathrm{~mm})$, (b) Length vs Power graph.

In order to solve this problem, we propose a novel serpentine structure with U-shaped bends in the arms of the MZI (see Fig. 4). The Ushaped bend configuration greatly increases the interaction length thereby enhancing the sensitivity of the sensor. This also allows high radius of curvature configurations reducing bend losses.

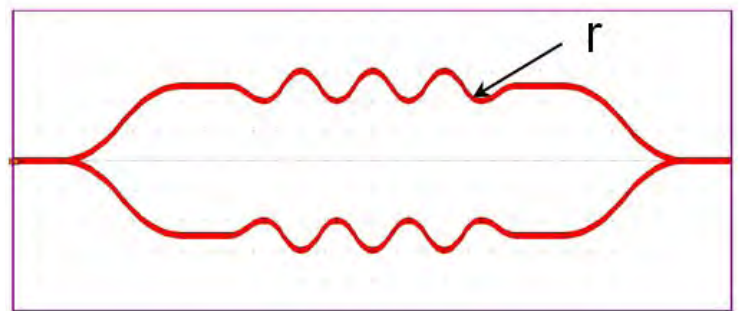

Fig. 4. Serpentine structure with U-bends of radius $r=28 \mathrm{~mm}$.

\section{Fabrication}

Fabricating optical biosensors based on silicon technology has been extensively studied for years. It mainly consists of a silicon substrate with silicon nitride (refractive index 2) as the core layer and silicon dioxide (refractive index 1.46) as lower and upper cladding layers. This configuration gives a high difference in core and cladding refractive indices which has a direct effect on improving sensitivity of the waveguide $[11,12]$. Moreover, since both silicon nitride and silicon dioxide are transparent, light propagates through the waveguide without significant attenuation.
To ensure monomodal propagation of light through the $\mathrm{MZI}$, the core layer must have a height of $250 \mathrm{~nm}$ and the upper and lower cladding layers should be more than $1.5 \mu \mathrm{m}$ so that the cladding layer is sufficiently thicker than the core thickness in order to have low attenuation losses as already shown in [8]. The $\mathrm{MZI}$ configuration was etched on the silicon nitride core layer using a photolithographic step. A rib of height $4 \mathrm{~nm}$ and $4-7 \mu \mathrm{m}$ width [4, 7] has been used for fabricating our sensor.

$\mathrm{MZI}$ with ribs of $4 \mathrm{~nm}$ height and varying widths such from 4 to $7 \mu \mathrm{m}$ were fabricated. Such low aspect ratio features possess inherent complications in fabrication. The fabrication was done on prime grade silicon wafers and consisted of twelve steps. The steps are designing and printing of the mask for photolithography, piranha cleaning of the silicon wafers, thermal oxidation of silicon wafer, LPCVD of $\mathrm{Si}_{4}$, photolithography, RIE, resist stripping, PECVD of $\mathrm{SiO}_{2}$, another photolithographic step using the same mask followed by RIE and resist stripping, and finally dicing (as shown in Fig. 5). All the fabrication steps were done in the NanoFab facility at the University of Alberta.

The second photolithographic step was performed in order to etch $1 \mu \mathrm{m}$ of the upper cladding layer, keeping the height of the core layer intact, in order to increase the aspect ratio. This provides better visualization of the features on the chip during alignment using the CMOS camera (see Fig. 1).

\section{Atomic Force Microscopy and Ellipsometry Results}

The rib dimensions were measured using Atomic Force Microscopy (AFM) (see Fig. 6). These AFM measurements were done after the first RIE step. Part (a) of the Fig. 6 shows a 2-D image of a $50 \times 50 \mu \mathrm{m}$ area of the chip and part (b) is a section graph showing the height of each point across the 2-D image along the reference (red) line. The RMS roughness was also calculated over various sections of the chip before and after PECVD of the $\mathrm{SiO}_{2}$ upper cladding layer and it was found to be in the range of $0.5-0.6 \mathrm{~nm}$, which is acceptable. It can be noted that the sidewalls are vertical and fairly smooth. Figure 7 is a three dimensional representation of one of the rib structures $(7 \mu \mathrm{m})$ on the chip.

Ellipsometry was used for determining the thickness and the refractive indices of the $\mathrm{SiO}_{2}$ cladding layers and the $\mathrm{Si}_{3} \mathrm{~N}_{4}$ core layer. The thermally grown $\mathrm{SiO}_{2}$ layer revealed a thickness of $1.3525 \pm 0.0007 \mu \mathrm{m}$. The refractive index was 
found to be $1.4668 \pm 0.0012$ which was close to the target refractive index of 1.46. The LPCVD nitride thickness was $252.6 \pm 0.3 \mathrm{~nm}$ with a refractive index of $2.0844 \pm 0.0051$ which was again close to the target of 2 . The upper cladding layer was found to be $1.9964 \pm 0.0030$ $\mu \mathrm{m}$ thick having a refractive index of $1.4631 \pm 0.0012$. a

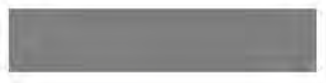

b
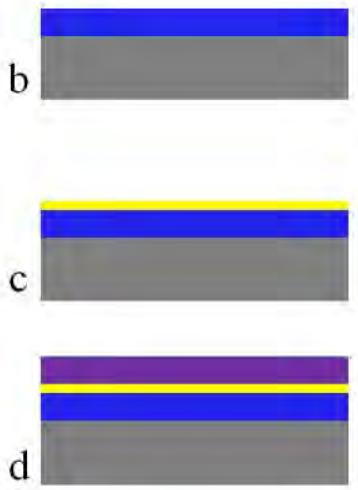

Silicon wafer

Silicon dioxide e

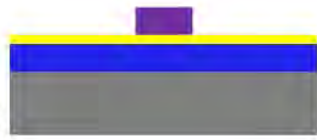

$\mathrm{f}$

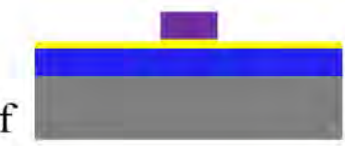

$g$

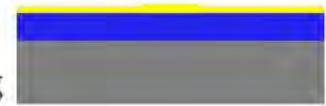

h

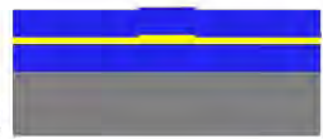

i

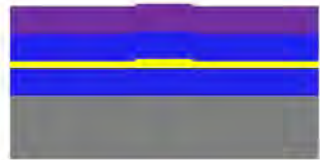

j

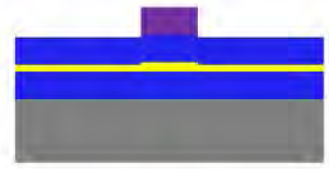

$\mathrm{k}$

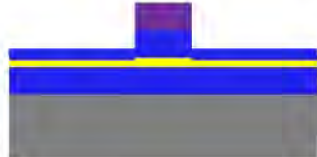

1

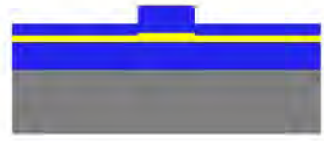

Silicon nitride

HPR504 (Photoresist)

Fig. 5. Schematic of the fabrication procedure. a. bare silicon wafer, b. thermal oxidation of silicon dioxide on the silicon wafer, c. LPCVD of silicon nitride, d. photoresist (HPR504) spinning on the silicon nitride layer, e. photolithography, $f$. RIE of $4 \mathrm{~nm}$ depth forming ribs, g. resist stripped from the rib, $h$. PECVD of silicon dioxide, $i$. HPR504 photoresist spinning on the silicon dioxide layer, j. photolithography, $k$. RIE of $1 \mu \mathrm{m}$ depth and I. resist stripped.
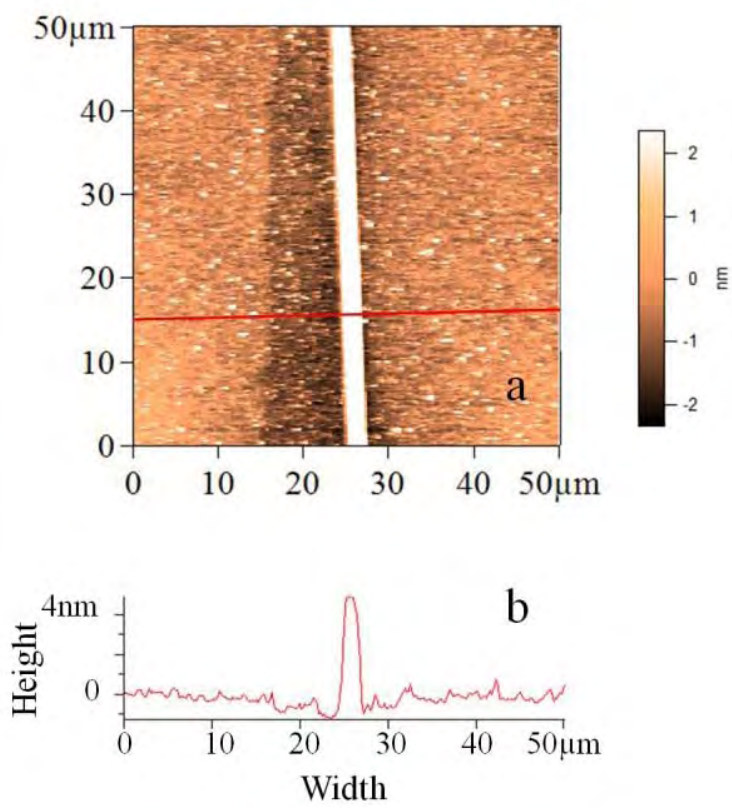

Fig. 6. (a) AFM image of a $4 \mu \mathrm{m}$ rib scanned over an area of $50 \times 50 \mu \mathrm{m}$; (b) Surface profile of $4 \mathrm{~nm}$ height rib along the reference (red) line shown in part (a).

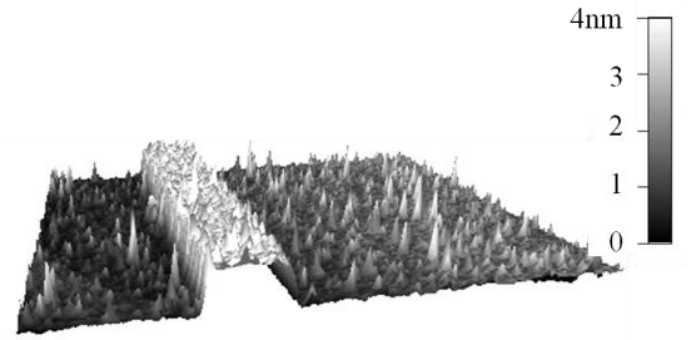

Fig. 7. Three-dimensional AFM image of a $7 \mu \mathrm{m}$ wide rib showing vertical $4 \mathrm{~nm}$ sidewalls.

\section{Conclusions}

We have shown the use of optical power loss analysis in simulations to design an MZI chip for use in biosensing applications. The fabrication procedure for nanodimensions based on silicon technology has been optimized. A novel design for high interaction length $\mathrm{MZI}$ devices has been proposed.

Future work involves improvement of the serpentine structure for achieving higher sensitivities. 


\section{Acknowledgements}

The financial supports from NSERC-CRD program and $A Q L$ Inc. are highly appreciated. We also gratefully acknowledge the valuable inputs from Stefania Dante, PhD student at Research Center on Nanoscience and Nanotechnology(CIN2), Spanish National Research Council (CSIC), Barcelona, Spain.

\section{References}

[1] R. Bashir, BioMEMS: state-of-the-art in detection, opportunities and prospects, Advanced drug delivery reviews 56, 1565-1586 (2004); doi: 10.1016/j.addr.2004.03.002

[2] Ph.M. Nellen, W. Lukosz, Integrated optical input grating couplers as direct affinity sensors, Biosensors and Bioelectronics 8, 129-147 (1993); doi: 10.1016/0956-5663(93)85025-J

[3] O. Parriaux,G.J. Veldhuis, Normalized analysis for the sensitivity optimization of integrated optical evanescent-wave sensors, Lightwave Technology, Journal of 16, 573-582 (1998); doi: 10.1109/50.664066

[4] F. Prieto, B. Sepúlveda, A. Calle, A. Llobera, C. Domínguez, A. Abad, A. Montoya, L. M. Lechuga, An integrated optical interferometric nanodevice based on silicon technology for biosensor applications, Nanotechnology 14, 907 (2003); doi:10.1088/0957-4484/14/8/312

[5] P. Englebienne, A. V. Hoonacker, M. Verhas, Surface plasmon resonance: principles, methods and applications in biomedical sciences, Spectroscopy 17, 255-273 (2003); doi:10.1155/2003/372913
[6] V. Koubova, E. Brynda, L. Karasova, J. Škvor, J. Homola, J. Dostalek, P. Tobiška, J. Rošický. Detection of foodborne pathogens using surface plasmon resonance biosensors, Sensors and Actuators B: Chemical 74, 100-105 (2001); doi: 10.1016/S0925-4005(00)00717-6

[7] E.F. Schipper, A.M. Brugman, C. Dominguez, L.M. Lechuga, R.P.H. Kooyman, J. Greve, The realization of an integrated Mach-Zehnder waveguide immunosensor in silicon technology, Sensors and Actuators B: Chemical 40, 147-153 (1997); doi: 10.1016/S0925-4005(97)80254-7

[8] R.G. Heideman, P.V. Lambeck, Remote optochemical sensing with extreme sensitivity: design, fabrication and performance of a pigtailed integrated optical phase-modulated MachZehnder interferometer system, Sensors and Actuators B: Chemical 61, 100-127 (1999); doi: 10.1016/S0925-4005(99)00283-X

[9] L.M. Lechuga, K. Zinoviev, L. Fernandez, J. Elizalde, O. E. Hidalgo, C. Dominguez, Biosensing microsystem platforms based on the integration of Si Mach-Zehnder interferometer, microfluidics and grating couplers, Proc. of SPIE 7220, 72200L (2009); doi: 10.1117/12.809166

[10] R.R. Syms, J.R. Cozens, Optical guided waves and devices, London: McGraw-Hill, (1992)

[11] W. Lukosz, Principles and sensitivities of integrated optical and surface plasmon sensors for direct affinity sensing and immunosensing, Biosensors and Bioelectronics 6, 215-225 (1991); doi: 10.1016/0956-5663(91)80006-J

[12] K. Tiefenthaler, W. Lukosz, Sensitivity of grating couplers as integrated-optical chemical sensors, JOSA B 6, 209-220 (1989); doi: 10.1364/JOSAB.6.000209 